\title{
Tecnologías Sociales y Economía Solidaria. Una revisión desde las realidades nacionales marcadas por la presencia de pueblos originarios ${ }^{1}$

\author{
Nicolás Gómez Núñez ${ }^{2}$
}

\author{
Categoría: Ponencia \\ Fecha de recepción: 15 de mayo de 2012 \\ Fecha de aprobación: 27 de junio de 2012
}

\begin{abstract}
Resumen
El escrito fue hecho durante y después de la participación en el IV Festival de Tecnologías Sociales y Economía Solidaria, que se realizó en la Universidad Federal de Rio de Janeiro en octubre de 2011. En su primera parte, el escrito presenta la categoría: posesión simbólica, para fijar las relevancias de las tecnologías sociales en las sociedades que habitan el medio social urbano pobre.

En la segunda parte, el escrito desenvuelve un diálogo desde las categorías de territorio, tecnología social y comunidad, con la descripción de las políticas públicas que promueven la Economía Social, las Tecnologías Sociales y la Economía Solidaria en Bolivia, Ecuador y Perú.
\end{abstract}

Palabras clave: Economía Social, Economía Solidaria, Pueblos Originarios, Buen Vivir, Tecnología Social.

\begin{abstract}
The paper was made during and after participation in the IV Festival of Social Technologies and Supportive Economy, held at the Federal University of Rio de Janeiro in October 2011. In its first part, the paper presents the category: symbolic possession, to settle the relevant aspects of social technologies in societies that inhabit the urban poor social environment.

In the second part, this article unfolds from the categories of land, social and technology community, with the description of public policies that promote the Social Economy, Social Technologies and Supportive Economy in Bolivia, Ecuador and Peru.
\end{abstract}

Keywords: Social Economy, supportive Economy, Indigenous Peoples, Good Living, Social Technology

\footnotetext{
1 Ponenciapresentadaenlamesade"EconomiaSolidáriaem realidadesnacionaismarcadas pela presença de povos originários, en el seminario: “AECONOMIASOLIDÁRIANAAMÉRICA LATINA - REALIDADES NACIONAIS E POLÍTICAS PÚBLICAS”, realizado por la Secretaría Nacional de Economía Solidaria (SENAES) del Ministerio del Trabajo y el Empleo de Brasil, en asociación con el Núcleo de Solidaridad Técnica (SOLTEC) de la Universidad Federal de Río de Janeiro (UFRJ), y con la Red de Investigadores Latinoamericanos de Economía Social y Solidaria (RILESS), llevado a cabo entre el 26 y el 28 de octubre de 2011.

2 Sociólogo (UAHC), Magíster en Desarrollo Humano a escala Local y Regional (IDERUFRO) y Doctor en Ciencias Sociales (FLACSO-Argentina). Académico e investigador de la Escuela de Sociología de la Universidad Católica Silva Henríquez, Chile.ngomezn@ucsh.cl
} 
Tecnologías Sociales y Economía Solidaria. Una revisión desde las realidades nacionales marcadas por la presencia de pueblos originarios - Nicolás Gómez

\section{Introducción}

Este artículo resulta de la invitación que hiciera el Núcleo de Solidaridad Técnica de la Universidad Federal de Rio de Janeiro, SOLTEC-UFRJ, a la comunidad académica de la universidad en la cual me desempeño. Al enterarme, solicité ser parte del debate sobre: "Economía solidaria en realidades nacionales marcadas por la presencia de pueblos originarios". Este interés se sostiene en dos razones.

La primera es que me encontraba buscando hechos que me permitieran comprender si el proceso de "desposesión simbólica" que había registrado Wacquant (2007), contribuía a explicar el papel del Estado en el fomento o en la destrucción de las posesiones simbólicas. Entre ellas, las tecnologías sociales que instituyen lo económico en el medio urbano pobre.

Esto es importante porque el Estado pierde fuerza en el análisis de Wacquant, aun cuando sí queda consignado en sus descripciones etnográficas que le permiten usar la noción de "parias urbanos" (Wacquant, 2007:129). Y así como él insiste en distinguir y separar a los "parias urbanos" de los Estados Unidos de Norte América en relación a los de Francia, yo tenía antecedentes suficientes para asumir que la categoría no era eficiente en Chile.

Uno de esos antecedentes es el proceso que logra hilvanar la identidad colectiva al fragor de las demandas por la vivienda ${ }^{3}$, donde la noción de "poblador" devela los clivajes de la disputa en el sistema político. Entonces, si siguiéramos el ejercicio hecho por Wacquant en las "poblaciones" de Chile, en las "favelas" de Brasil, en las "villas" de Argentina, en las "barriadas" de México o en los "pueblos jóvenes" de Perú, habría una alta probabilidad de observar que el Estado desempeña un papel crucial en las tecnologías sociales.

3 En este mismo sentido, Lomnitz (2008:113-114), siguiendo a Manuel Castells, confirma que los movimientos sociales en América Latina se caracterizan por la demanda de la vivienda mediante asentamientos urbanos. Por tanto, son uno de los principalescanalesdeparticipaciónpolíticaydeorganizacióncomunitariaenlaciudad. 
En el caso de la comuna de Huechuraba, ubicada en la Región Metropolitana de Chile, la cual emerge gracias al asentamiento de 3.000 familias aproximadamente, entre 1969 y 1973 (Gracés, 1997:108-109), una posible lectura de su historia puede resumirse como a continuación se presenta.

Una vez que se acumularon las demandas por un techo donde habitar, los "sin casa" y "allegados" emprendieron la "toma" de un terreno en contra del Estado y de sus propietarios. Para los "sin casa" y "allegados", esa "toma" no sólo implicó conquistar un terreno. Además supuso co-habitar hitos de arraigo en un tiempo que fue excepcional porque ahí estuvo la reciprocidad colectiva donde se llevaron a cabo las combinaciones de todos los recursos disponibles para lograr bienes y servicios apropiables por ellos (Salazar, 2006: 111; Hardy, 1985:27), y se instituyeron los arreglos sociales que produjeron la seguridad colectiva.

Por tanto, fue en ese tiempo histórico donde se desarrolló esa "sociedad popular" (Salazar, 2000:18), es a partir de ahí que la categoría "poblador" reportó la síntesis de la posición de cada uno de los individuos en la trayectoria de la reciprocidad que reproduce el modo de hacer las cosas o la tecnología que les es inherente a ellos, y justificó la reivindicación de los bienes de uso comunitario de la sociedad popular que se instituyó. Entonces, la categoría "poblador" es la posesión simbólica de ese "colectivo social de cara al futuro" ${ }^{4}$ " capacitado para rebasar el marco de las identidades históricas" (Salazar, 2000:13).

\footnotetext{
4 “¿Qué ocurría en la población en los días previos al golpe? Se vivía un ambiente de mucha vitalidad, se vivía con mucha fuerza el despertar popular. Ser obrero, ser poblador en ese tiempo tenía un peso en la sociedad. Eso fue lo que más me impactó, no es que no existieran problemas, contradicciones, sectarismos, errores, corruptelas. Pero era un pueblo que se ponía de pie. No para tomar las armas, como inventaron otros para justificar lo que vino después, sino para enfrentar su propio destino, para enfrentar su problema de salud, de convivencia en la cuadra, de abastecimiento, sus problemas laborales.

Estaba todo sembrado de organizaciones populares y la gente crecía a pasos agigantados en dignidad, en conciencia, en compromiso, en solidaridad. No era esa gente pasiva, resignada, fatalista que yo había conocido en visitas a las poblaciones en años anteriores" (Extracto de entrevista a Ronaldo Muñoz sscc, en Pastor, et al., 1993:134).
} 
Tecnologías Sociales y Economía Solidaria. Una revisión desde las realidades nacionales marcadas por la presencia de pueblos originarios - Nicolás Gómez

Con el correr del tiempo los "pobladores" se relacionaron con organizaciones no pobladoras, contribuyendo a mantener un proceso de "democratización" (Garretón, 1990:19). Entre esas relaciones destacan las que amasaron el capital social político que logró situar sus demandas en las políticas públicas del Estado y de los municipios. Esta apertura del sector público estuvo inscrita en el período de la "Democracia Populista" (Salazar, 2006: 95; Grez y Salazar, 1999:14). Aquí Garcés (1997: 44-45) inscribe el Plan Habitacional del presidente Jorge Alessandri (1958-1964) como reacción a la "toma" de 1957, la que dio origen a la Población La Victoria en la zona sur de Santiago. Luego, ese proceder del Estado irá legitimándose como promoción de las organizaciones de los "pobladores" en el gobierno del presidente Eduardo Frei (1964-1970), a través de su "Operación Sitio"; para finalizar en el gobierno del presidente Salvador Allende (1971-1973).

Este despliegue de relaciones experimentó un nuevo tiempo social con el "golpe" de Estado del 11 de septiembre de 1973. Esta "acción faccionalista" (Grez y Salazar, 1999: 10) que expresa una "operación estratégica" en el marco de una "revolución neoliberal" (Salazar, 2006: 96), impulsó un proceso de desposesión simbólica gracias a la destrucción de las fuentes de legitimación de la identidad política y del trabajo de reproducción de las tecnologías sociales de los "pobladores". Luego, el Estado chileno puso en marcha los mecanismos de producción de los estigmas (Goffman, 2006:30-35, 45-47) que sustentan el control y la represión.

Por ejemplo, en el ámbito económico el Estado revivió la definición que se le daba al labrador de la primera mitad del siglo XIX. Por tanto, el "poblador" fue asumido como la "versión criolla del siervo de la gleba medieval" (Salazar, 2000:76): carente de iniciativa, flojo y vicioso por naturaleza, y cuando el estigma fue flexible se dejó caer el rótulo de "delincuentes".

Esta breve y gruesa descripción me permite sostener que las tecnologías sociales son mucho más que las relaciones que pro- 
ducen bienes y servicios de uso colectivo, en la medida que ellas expresan un tiempo social y una forma de estar en la vida. Por tales variables, éstas son un tipo particular de posesión simbólica que participa en el diseño e implementación de la política por parte de los grupos y comunidades que se encuentran en el extremo desigual del crecimiento económico, debido a que avanzan a pesar de la despreocupación del Estado.

La segunda razón que justifica mi interés por conseguir la invitación hecha por SOLTEC-UFRJ, es la siguiente. Los que trabajamos en proyectos sociales sabemos que muchas iniciativas son formuladas sin incorporar la opinión de los que se nombran como beneficiarios, población objetivo o población directa. En el caso que he expuesto, ese sesgo se expresaría como el intento por idear un proyecto asumiendo que en el medio social urbano pobre hay un desierto en materia de organización, y que no existen las tecnologías sociales.

Planteado así el asunto, acontece un desfase entre el equipo que implementa un proyecto y el grupo o las comunidades que constituyen la sociedad popular que participa en sus servicios; y ahí la noción de tecnología social nos permite ordenar dos reflexiones.

La primera se refiere a que la coherencia de los proyectos se logra gracias a un "diagnóstico operativo" (Forni, 1992:32) que describe, analiza e incluye las tecnologías sociales de la sociedad popular. Para lograr ese objetivo, hay que cambiar el desempeño de los equipos que ahí intervienen, y ese giro se refiere a un tránsito que abandona el modelo de capacitación técnica y profesional, el cual los convencía de ser los que "lideran" (Castells, 2002:157) el proceso de empoderamiento de los receptores de sus servicios; y adoptar el modelo de "calificación social" (Perret y Roustang, 2000:91) que los sitúa en una posición similar a la que tienen los que se asocian para poner en marcha iniciativas que colectivamente se han sancionado como pertinentes. 
Tecnologías Sociales y Economía Solidaria. Una revisión desde las realidades nacionales marcadas por la presencia de pueblos originarios - Nicolás Gómez

La segunda reflexión, constata la modificación en los supuestos de uso de los diseños de las investigaciones y diagnósticos gestados dentro de un proyecto. Dónde, por ejemplo, resulta pertinente asumir que las estrategias de sobrevivencia son "acuerdos básicos, socialmente definidos y concretamente aplicados" (Forni, Benenci y Neiman, 1991:77). Así, aprehender a desempeñarse en esas "reglas" (Winch, apud., García, 1994:94) o en esas "tradiciones" (Gadamer, apud., García, 1994:94) de la sociedad popular para adecuar los tiempos sociales (Bourdieu, 2006:62-65), al menos el de la implementación del proyecto y el de la vida cotidiana de los beneficiarios, supone abandonar las categorías que permiten representar a esos grupos y comunidades en la planificación ${ }^{5}$, especialmente cuando se la ha ideado como si existiese una cultura urbana homogénea (Castells, 1999:119-121).

Las razones que he descrito inspiran mi participación en este IV Festival de Tecnologías Sociales y Economía Solidaria, que se

5 En este sentido la obra de Herrera (2011) nos muestra cómo los buenos ánimos de recuperar la tecnología del pasado en los Andes, especialmente en Ecuador, Perú y Bolivia, va develando una imposición de formas ya definidas de obrar por quienes implementan los proyectos sociales sin tomar en cuenta que la tecnología social es mucho más que los artefactos que vemos a mano. A modo de ejemplo de esta reflexión, el siguiente extracto de Herrera.

“En la generación de los proyectos de desarrollo basados en la 'recuperación', la contratación de especialistas -con frecuencia, consultores independientes u ONGdesempeña un papel fundamental. Por lo general, se trata de especialistas con algún grado de instrucción superior formal, desligados de los contextos locales y, con frecuencia, extranjeros que vienen a conocer las realidades locales por intermedio de los mismos contratantes. Independientemente de su origen, existe el riesgo de que los consultores y ONG adopten -parcial o totalmente- las formas de ver y entender la historia y el entramado social local, prefiguradas por la situación de poder desde la cual actúan el Estado, la Iglesia, las ONG o el agente institucional en cuestión, sin mencionar la posible existencia de prejuicios propios. En casos en donde hay urgencia de ejecutar un proyecto, en la antesala de una elección o del término de programas de financiamiento multilaterales, el hecho de consultar a los actores locales puede pasar a segundo plano, incluso en proyectos de desarrollo participativo y, sobre todo, cuando hay resistencia local. Irónicamente, una menor consulta de la población local puede facilitar a los gerentes de proyectos la presentación 'exitosa' ante los entes financistas del desarrollo de la implementación de ideas generadas desde 'arriba'." (Herrera, 2011:130) 
realiza en la Universidad Federal de Rio de Janeiro, y me llevan a conocer lo que presenta Beatriz Delgado de Bolivia, Patricio Andrade de Ecuador y Humberto Ortiz de Perú. En ese conocer, me he permitido hacerles comentarios tomando en cuenta tres tópicos, a saber: territorio, tecnología social y comunidad.

\section{Territorio}

Indudablemente que buena parte de lo que hoy entendemos por territorio ha sido considerado por las Ciencias Sociales, especialmente cuando se lo interpreta como una pieza del andamiaje económico y de un modelo político. Por ejemplo, de la mano de Boisier (2001) podemos identificar la perspectiva del "desarrollo territorial", donde hay una apuesta por ese "recorte" de la "superficie" que "contiene" al "territorio organizado", el cual está dotado de una estructura administrativa.

Luego han sido las elucubraciones sobre las consecuencias "perversas de la modernidad", por citar el título de la compilación de Berian (1996) en la cual pone en juego las reflexiones de Giddens, Bauman, Luhmann y Beck, para trazar puentes entre las orillas de la diversidad que adopta el riesgo y la reflexividad en el proceso de individuación; o, en torno a las formas de salida de la globalización, las que han permitido indicar al territorio como un lugar donde suceden acontecimientos alternativos, innovadores y en no pocos casos, la idea de territorio ha sido transportada para fijar un lugar aparentemente seguro ${ }^{6}$ donde se pueden experimentar las capacidades de lo local y lo endógeno.

6 La búsqueda de la seguridad en una "modernidad sin modernismo" (Bauman, 2003:126), es una ocupación importante en la obra de Bauman: "Comunidad. En busca de seguridad en un mundo hostil". Sin embargo, el papel que él le asigna a lo local o al "lugar" (Ibid.: 131-132), es siempre desde la sospecha de la imposibilidad de que suceda de la manera como en este trabajo lo presento. 
Así las cosas, es posible suponer que los procesos que se dan en lo inmediato de la vida cotidiana y que reconocemos como el territorio, han caminado desde siempre constituyendo sus formas de hacer lo social y con independencia de la relevancia que le puedan haber asignado los investigadores. $\mathrm{O}$, que es lo mismo, frente a las inquietudes que plantea la globalización o los resultados no deseados del proceso de modernización, los investigadores se han volcado a observar qué es lo que puede ofrecer el territorio.

Desde otro abordaje y con los pies puestos en Latinoamérica, observo en la presentación que realiza Beatriz Delgado -la cual se ha concentrado en las organizaciones de la economía solidaria y el comercio justo-, la primera categoría que fija un lugar común con las exposiciones de Patricio Andrade y Humberto Ortiz: se trata del Ayni Minka.

Considerar esta concepción para discutir sobre el territorio es, como lo plantea Beatriz Delgado, avanzar por aquella diplomacia de lo pueblos que nos permite vernos como una comunidad. Esta postura, sin embargo, nos distancia de la posibilidad de "recortar" una "superficie", nombrarla y actuar sobre ella desde fuera. Entonces, el Ayni Minka contribuye a promover el argumento desde lo que hay dentro del territorio, especialmente desde las relaciones que instituyen el tiempo vital, ese que declara una trayectoria junto a todo lo que rodea la existencia.

Es en ese sentido que Humberto Ortiz nos conduce a pensar en una integración socioambiental, donde el supuesto es el reconocimiento de cada una de las expresiones de la biodiversidad. Por tanto, el territorio se visualiza como la energía de la reciprocidad que produce la redistribución de los bienes, y ese obrar natural se ubica como la ética de la vida comunitaria. Al decir de Patricio Andrade, ese marco del universo simbólico es el Sumak Kawsay (Buen vivir).

Este Sumak Kawsay es la matriz del Ayni Minka. En consecuencia, produce los grupos primarios de reciprocidad. Así se ex- 
presa en la familia relacionada por los vínculos consanguíneos y de parentesco, y en la familia fundada en la familiaridad del "comunitarismo indígena" (García, 2004:439) que se logra cuando las relaciones permiten que cada persona sea más que un conocido, porque ellas son parte de la rememoración de la experiencia, están en el diseño del futuro y a través de ellas es probable crear y usar los bienes colectivos, locales o comunales.

Entonces la palabra territorio intenta traducir esas relaciones de reciprocidad y distribución que están dispuestas en un sentido trascendente, y donde es posible indicar hitos de arraigo de la convivencia. Esto es, finalmente, lo que define la identidad de la relación que cada persona arrastra consigo y que reproduce colectivamente, o donde realiza el buen vivir según Humberto Ortiz.

Un ejemplo de este tiempo de reciprocidad y distribución se encuentra en las ceremonias donde se realizan los sacrificios y las ofrendas a los espíritus de los muertos, a los espíritus de la naturaleza y a las fuerzas mayores. Ahí participa la comunidad, los artefactos que representan a las divinidades, los que permiten exhibir lo ofrecido, y también las localidades como los Apus (Montañas Sagradas).

Para el comentario que realizo, esas ceremonias son relevantes porque manifiestan con mucha claridad el doble vínculo de la reciprocidad y de la distribución. Uno de esos vínculos es la inclusión de cada individuo a su comunidad y de la participación de la comunidad hacia y en cada integrante de ella; el otro vínculo es el de la comunidad con las divinidades y de las divinidades con la comunidad.

Entre esas relaciones, el Sumak Kawsay muestra toda su institucionalidad cuando orienta a los hombres y a las mujeres a definir sus relaciones con las divinidades desde la "prestación total" (Mauss, Op. cit. Godelier, 1998: 62). En consecuencia, ellos están gobernados por una concepción que obliga a una colectividad, y no sólo a un individuo, y lo hace de manera perpetua, 
Tecnologías Sociales y Economía Solidaria. Una revisión desde las realidades nacionales marcadas por la presencia de pueblos originarios - Nicolás Gómez

abarcando a los que ya no están presentes físicamente, a los que habitan circunstancialmente en los ríos, lagos, en la cordillera de Los Andes, a los que físicamente participan en las tecnologías sociales que producen la ceremonia de ofrenda, y a los que vendrán en un futuro que es solicitado en esas rutinas reglamentadas de común unión.

Uno de los resultados relevantes de este tipo de relaciones de reciprocidad y distribución entre comunidades y divinidades, es que facilita la existencia de un ámbito autónomo dentro de la cultura, en el cual no sólo se elucubran razones distantes de las que hilvanan el valor comúnmente monetario de las mercancías ${ }^{7}$, sino que obra en resistencia y conflicto con las racionalidades del modelo neoliberal. Más aún cuando este modelo se proclama como la única alternativa capaz de renovar el contrato entre los integrantes de la comunidad y entre ellos y las divinidades, y ejerce con sus tecnologías sociales un proceso de desposesión simbólica que intenta imponer, a como dé lugar, la explotación para lograr utilidades.

Frente a ese allanamiento del modelo neoliberal, en donde tradicionalmente el Estado se ha mostrado proclive a jugar a su favor, la institución del Sumak Kawsay predispone a la asistencia en la emergencia y a la defensa del árbol que cae, a la protesta por el lago que es contaminado por los desechos industriales, a la barricada que impide que las máquinas talen el bosque, a la mar-

7 Con el objetivo de clarificar las dimensiones que se encuentran en juego en esta tensión entre dos cosmovisiones, consigno la descripción que Godelier hace sobre el sistema capitalista. "Si el sistema capitalista es complejo en sus estructuras, es simple en sus principios []. El sistema capitalista se presenta como el sistema de producción de mercancías históricamente más desarrollado de todos los que han existido. Se basa en el principio de la propiedad privada de los medios de producción, de los medios de consumo y del dinero, así como en la venta y compra de la fuerza de trabajo intelectual y/o manual que interviene en los procesos de producción y de circulación de las mercancías. Su resorte es simple: el deseo de producir dinero con el dinero, lo que implica transformar el dinero en capital e invertirlo en proceso de producción y de circulación de las mercancías []." (Godelier, 1998:96) 
cha a favor de las personas a las cuales se les arrebata su derecho a participar en los beneficios del contrato de reciprocidad y distribución, porque el Sumak Kawsay ayuda a ver en cada uno de ellos a los espíritus y sus poderes; y sólo después, como sostiene Godelier (1998:176), se los ordena como símbolos y signos de ese pacto entre la comunidad y las divinidades ${ }^{8}$.

Por lo antes dicho, cabe una conclusión preliminar. La concepción de territorio se devela gracias a las múltiples piedras angulares que provienen de una misma matriz, la cual es el Sumak Kawsay; y esas piedras angulares son los contratos que hilvanan la historicidad Ayni Minka de la comunidad. En ese proceso de reproducción y representación, convergen relaciones que son, al miemos tiempo, "sociales, intelectuales y afectivas, y que se materializan en objetos" (Godelier, 1998:194-195). Por lo tanto, en el único mundo posible para la comunidad, el territorio es un tipo

8 En esta expresión del Sumak Kawsay, se observa explícitamente un fenómeno identificado por Mauss, a saber: el hecho social total, y según Godelier, el asunto se resume de la siguiente forma: "A partir del momento en que, en una sociedad, la mayor parte de las relaciones sociales existe sólo bajo la forma y gracias a la instauración de vínculos personales, de relaciones de persona a persona, a partir del momento en que el establecimiento de vínculos pasa por el intercambio de dones que, a su vez, implican transferencias y desplazamientos de "realidades" que pueden ser de todo tipo [] mientras puedan ser objeto de una distribución; todas las relaciones sociales objetivas que forman los cimientos de una sociedad [], así como las relaciones personales e intersubjetivas que las encarnan, pueden expresarse y "materializarse" en dones y contradones, así como en los desplazamientos, trayectos, que efectúan los "objetos" de esos dones.

Y debido a que el don, en tanto acto de donar, en tanto donación, en tanto práctica real, es un elemento esencial de la producción-reproducción de las relaciones sociales objetivas y de las relaciones personales, subjetivas e intersubjetivas, que son su modo concreto de existencia, el don en tanto práctica forma parte simultáneamente de la forma y del contenido de esas relaciones. En ese contexto precisamente, el don, en tanto acto pero también como objeto, puede re-presentar, significar y totalizar el conjunto de las relaciones sociales del que es a la vez instrumento y símbolo. Y en la medida que los dones proceden de las personas y los objetos donados se vinculan primero para luego liberarse y volver de nuevo a vincularse a las personas, los dones encarnan tanto a las personas como a sus relaciones. En este sentido y por estas razones, el don -como lo expresó con maestría Mauss- es un "hecho social total": en tanto contiene y une a la vez algo que procede de las personas y algo que está presente en sus relaciones, consigue totalizarlas y simbolizarlas en su práctica y en los objetos que la materializan" (Godelier, 1998:154). 
Tecnologías Sociales y Economía Solidaria. Una revisión desde las realidades nacionales marcadas por la presencia de pueblos originarios - Nicolás Gómez

especial de objeto, en la medida que realiza la "síntesis de lo real y lo imaginario, que son los componentes del ser social" (Godelier, 1998:197) de la comunidad.

A continuación es conveniente responder la siguiente cuestión: ¿Qué similitudes y diferencia puede tener esta concepción de territorio, con otras que se utilizan en las políticas públicas que hoy escuchamos?

De las tres ponencias, observo que la de Patricio Andrade nos plantea con claridad el asunto de la unidad en la diversidad, lo cual nos lleva a figurar un territorio donde su estilo de desarrollo se define por la coordinación de las formas económicas. Por ejemplo: las que se dan entre la economía del sector público, privado y popular; la de las redes de redes y los circuitos económicos de economía social y popular.

En ese contexto y a modo de ejemplo, Patricio nos ha nombrado la "eco tienda" "De la mata a la olla" (www.delamataalaolla. wordpress.com), donde hay dos objetivos: desarrollar las comunidades rurales desde la economía solidaria y el comercio justo, y producir hortalizas libres de químicos.

Esta eco tienda, que nace en un grupo de la comunidad de Quito, incluye a los consumidores y productores, por lo que la cadena de comercialización se torna justa y humana, y reduce la participación de los intermediarios. Luego, y esto es lo que a mí me llama la atención para la reflexión sobre el territorio, la iniciativa ha logrado una coordinación que forja un tejido de relaciones cara a cara y también de manera virtual, mediante lo cual brinda -siguiendo textualmente a Forni (1992:14)- servicios de transferencia de recursos, producción, distribución, capacitación, organización, intervención y construcción de sistemas de interacción entre nuevos actores sociales.

Esta forma de entender la producción y el consumo, supone la existencia de un "circuito de satisfacción de necesidades", en cuyo centro están las "necesidades de sociabilidad", a saber: la necesidad de "consideración", "dignidad", "autoestima", la necesidad de te- 
ner un sentido de existencia y de dotar de sentido al mundo (Forni, 1992:14). Y este circuito se observa posible, gracias a la participación de las organizaciones que están en un mismo territorio, especialmente porque el Estado no sólo aplaude las iniciativas de la comunidad, sino que también se vuelca con recursos para ser uno más ${ }^{9}$.

A mi entender, el ejemplo presentado por Patricio es una muestra de la compatibilidad entre la propuesta del Ayni Minka y la Teoría del Desarrollo Endógeno y Local (TDEL). Especialmente porque ambas comparten concepciones que definen el pensar y permiten el hacer.

La primera de estas concepciones es la noción de red, la que se ocupa para nombrar al conjunto de vínculos inscritos en un obrar histórico definido. Luego, estimo que si una parte de esa red contribuye a producir bienes y servicios apropiables por la comunidad, entonces estamos frente a la tecnología inherente a ese colectivo. Así, el asunto central es que dichos sistemas organizados históricamente son los que facilitan la integración y explican las posibilidades de crecimiento económico gestado desde el nivel local.

A continuación podríamos sostener que la posibilidad de sostener ese desempeño se produce, en tanto la comunidad amplía las relaciones con grupos con tecnologías sociales diversas. Sin embargo, aparece una distancia cuando la TDEL plantea que el "centro" es un límite que circunscribe las relaciones y las funciones de integración y de crecimiento, a diferencia de la noción de territorio que se desprende del Ayni Minka. Pero esta diferencia se reduce cuando constatamos que el Estado ha implementado procesos de desposesión simbólica.

9 Según el artículo cuarto del documento constitucional que crea el Instituto Nacional de Economía Popular y Solidaria de la República de Ecuador, alojado en el Ministerio de Inclusión Económica y Social, este debe promover la vinculación y cooperación de los distintos actores locales y nacionales para expandir la economía popular y solidaria. 
Al respecto, García nos ofrece una descripción que ejemplifica lo señalado.

A diferencia de lo que sucedió desde los años treinta del siglo XX, cuando los movimientos sociales fueron articulados en torno al sindicalismo obrero, portador de un ideario de mestizaje, y resultante de la modernización económica de las elites empresariales, hoy los movimientos sociales con mayor poder de interpelación al ordenamiento político son de base social india, emergentes de las zonas agrarias, bloqueadas o marginadas de los procesos de modernización económica impulsados desde el Estado.

Los aimaras del altiplano, los cocaleros de los Yungas y el Chapare, los ayllus de Potosí y Sucre, y los indígenas del oriente han desplazado en el protagonismo social a los sindicatos obreros y organizaciones populares urbanas. Y, a pesar del carácter regional o local de sus acciones, comparten una misma matriz identitaria indígena, que interpela el núcleo invariante del Estado boliviano desde hace 178 años: su monoetnicidad. El Estado boliviano, en cualquiera de sus formas históricas, se ha caracterizado por el desconocimiento de los indios como sujetos colectivos con prerrogativas gubernamentales. Y el hecho de que hoy aparezcan los indios, de manera autónoma y como principal fuerza de presión demandante, pone en cuestión, precisamente, la cualidad estatal, heredada de la colonia, de concentrar la definición y control del capital estatal en bloques sociales culturalmente homogéneos y diferenciados de las distintas comunidades culturales indígenas que existieron antes de que hubiera Bolivia, y que, incluso ahora, siguen constituyendo la mayoría de la población (García, 2004:430). 
La segunda noción importante en la TDEL es la de capital cultural -yo creo que esa es la variable que Bourdieu (2006) devela en los procesos de desposesión simbólica-, la cual especifica la idiosincrasia que está en las redes y que en definitiva gobierna su orientación. Para el caso en que nos encontramos, el capital cultural es el Sumak Kawsay, y sus códigos socioculturales gobiernan la elaboración de un saber sobre la realidad y definen la forma de atribuir ese conocimiento, especialmente en las técnicas y procedimientos de las relaciones de reciprocidad y distribución.

Para otros investigadores como Boisier, la formulación de la TDEL goza de cuatro niveles. El primero es el político, y su desempeño eficiente será evaluado en la medida que el territorio organizado: región o localidad, cuenta con la capacidad para tomar decisiones sobre su desarrollo. Un segundo nivel es la capacidad que tiene el territorio para apropiarse y reinvertir parte del excedente que genera su modelo económico, y es aquí donde Boisier fija la diversificación de la estructura productiva, porque él entiende que de esa manera es posible la sustentabilidad. El tercer nivel es la capacidad que tiene el territorio para gestar un modelo de producción de conocimiento y de asignación de ese saber hacer, o, como sostiene Boisier, se trata del plano científico y tecnológico. $Y$, en el último nivel encontramos la "matriz generadora de la identidad socioterritorial", la cual nombra como plano cultural (Boisier, 2006:13-14).

Por otro lado, la lógica de la TDEL también asume que son las relaciones entre los componentes, y no los componentes de esas relaciones: personas, organizaciones $\mathrm{u}$ artefactos, los que definen y facilitan la reproducción de la sociedad; y en su comprensión apela a modelos circulares basados en flujos de conocimiento y en las capacidades asociativas.

En este sentido, es bastante gráfica la siguiente expresión de Boisier:

[] el desarrollo endógeno puede ser entendido como una propiedad emergente de un sistema territorial que 
posee un elevado stock de capitales intangibles y sinergéticos,[]. En otras palabras, el desarrollo endógeno se produce como resultado de un fuerte proceso de articulación de actores locales y de variadas formas de capital intangible, en el marco preferente de un proyecto político colectivo de desarrollo del territorio en cuestión.

Todo proceso de desarrollo endógeno se vincula al desarrollo local de una manera asimétrica: el desarrollo local es siempre un desarrollo endógeno, pero éste puede encontrarse en escalas supra locales, como la escala regional por ejemplo. (Boisier, 2006:14)

Otra similitud entre la propuesta del Ayni Minka y la TDEL, es la forma de llevar a cabo el proceso de gobierno del desarrollo. Así, la vocación política es trasladar al nivel local la toma de decisiones que articulan el diseño, la implementación y la evaluación de la política pública. Por ejemplo, y siguiendo a García, se trataría de una marcha donde los "bloques sociales excluidos" politizan lo social a través de un nuevo tejido de redes con capacidad de imponer una mira sobre el destino de su sociedad, desarticulando la estrategia neoliberal de individuación de las "clases subalternas, revirtiendo lo pactado en términos de privatización de los bienes comunes y reintegrando los elementos de la vital existencia, como el agua (García, 2006: 429-429).

Desde estas semejanzas podemos asumir que tanto la Teoría del Desarrollo Endógeno y Local como la basada en el Ayni Minka, ponen en cuestión el principio que supone una evolución de lo social, especialmente si las etapas a ser superadas están dadas de antemano por un tipo ideal de sociedad. Así, ambas asumen la existencia de diversos modelos de sociedad que pueden ser horizontes de inspiración.

Este factor es una de las dimensiones que nos permitiría comprender los cuestionamientos y las crisis a nivel de las "estructuras de invariancia estatal" y en las "estructuras estatales 
temporales" ${ }^{\prime 10}$, que viven los Estados que se ubican en la posición neoliberal, por ejemplo el "Estado neoliberal-patrimonial" (García, 2006:427), y que han sido impermeables a las emergencias de las demandas por participación reclamadas por las sociedades populares, o, como el caso chileno, que han profundizado la herencia constitucional heredada por las dictaduras.

Para cerrar este apartado podemos señalar que el territorio no es sólo un factor productivo y administrativo, porque cuando situamos nuestra atención desde lo que los pueblos originarios enseñan, la categoría abarca dos niveles que trascienden esas apreciaciones. El primero comprende la proximidad social que sostiene a las comunidades que producen bienes y servicios de uso colectivo; y el segundo nivel es un tiempo al que le es inherente un contenido denso que orienta las proximidades e instituye las relaciones de cooperación y distribución.

\section{Tecnología Social}

A continuación abordaré la noción de tecnología social dentro del Ayni Minka, para lo cual reconozco un criterio de demarcación que se encuentra en las tres presentaciones que hoy hemos escuchado. Este criterio se refiere a que la tecnología social se inclina hacia su función de integración reglamentada dentro de un territorio, o, como sostiene García parafraseando a Habermas, desde la apelación a la "acción normativa" en desmedro de la "acción comunicativa" (García, 2004:345).

10 "La primera tiene que ver con un deterioro radical y un cuestionamiento de las certidumbres societales, institucionales y cognitivas que atraviesan de manera persistente los distintos ordenamientos estatales de la vida republicana, a las que llamaremos estructuras de invariancia estatal; mientras que la crisis de "corta duración" hace referencia al modo "neoliberal" o reciente de configuración del Estado, al que llamaremos estructuras estatales temporales que, pese a sus variadas formas históricas, utilizan, moldean y dejan en pie sistemas de poder que dan lugar a las estructuras invariantes. Veamos brevemente cómo se manifiesta esto." (García, 2006:427). 
Luego la tecnología social puede ser conceptualizada recurriendo a dos nociones que reflejan cursos de acción. La primera, como ya lo consignamos, es la red de vínculos con la historia de los obrares colectivos que produce bienes y servicios apropiables por la comunidad.

El asunto central es que dichos sistemas organizados son los que permiten la integración en base al trabajo. Pero aquí, en el Ayni Minka, el trabajo válido y socialmente aceptado es aquel que en su desempeño instituye lo público desde la relación entre la familia, la comunidad y la naturaleza, por ende, el trabajo comunitario es el que sintetiza el buen vivir de esa convivencia.

Es por tal razón que Beatriz Rodríguez declara una distancia entre este trabajo comunitario del Ayni Minka y la que nosotros podemos apreciar en el Mercado de Trabajo, conformado no sólo pero sí básicamente, por la compra de fuerza de trabajo mediante un salario y donde los beneficios, tanto del que compra como del que vende, quedan en el ámbito individual.

Por otro lado, esta definición de tecnología social no debe confundirse con la categoría de capital social. Según Durston, el capital social puede ser entendido de la siguiente forma.

[] se entenderá por capital social el contenido de ciertas relaciones y estructuras sociales, es decir, las actitudes de confianza que se dan en combinación con conductas de reciprocidad y cooperación. Ello constituye un capital en el sentido de que proporciona mayores beneficios a quienes establecen este tipo particular de relaciones y que puede ser acumulado (Durstron, 2009:1).

La diferencia entre la tecnología social y el capital social, radica en que la primera es un sistema de relaciones que ya cuenta con un reglamento, el cual podrá variar por razones objetivas que emergen desde los contenidos de los vínculos de la comunidad que instituye la tecnología social cada vez que la necesita. Por el 
contrario, el capital social es un vínculo diádico, su mejor expresión es el compadrazgo, y si bien lo podemos encontrar cuando liga las familias que coexisten en un mismo territorio: barriada, población, favela o villa, escasamente involucra a la comunidad que habita el territorio.

Otro elemento que distingue a la tecnología social y al capital social, tiene relación con que la primera es un modo de hacer que involucra una manera de construir conocimiento, y una forma de usar socialmente ese saber. Por su parte, el capital social se articula en la emergencia colectiva por la obtención de un recurso y una vez que se ha logrado, ese recurso permitirá concretar un objetivo socialmente aceptado. De esta forma, el capital social puede ser parte -a veces es una parte crucial debido a la energía desplegada por la búsqueda del recurso-, de la tecnología social.

A continuación nombraré las cualidades que dibujan la forma y el contenido de la tecnología social que hemos visto. Indiscutiblemente que varios de esos aspectos yo los he exagerado para expresarlos como si fueran parte de un tipo ideal. Entonces, lo que diré no podrá ser visto con esa explicitación en la realidad. Sin embargo, este ejercicio me parece oportuno porque al resaltar esas características, podemos contar con espacios definidos para el juego del debate y para que florezcan búsquedas que sólo son posibles a partir de la conversación sobre cada uno de nuestros planteamientos.

Entonces, la tecnología social puede describirse tomando en cuenta cinco características, a saber:

1. La tecnología social cuenta con un saber hacer orientado al cumplimiento de uno o varios objetivos compartidos por quienes instituyen un tiempo social desde las evaluaciones sobre la trayectoria de la convivencia.

Este saber hacer está organizado por las combinaciones de las informaciones sobre los usos reales y potenciales de lo que está a mano en la vida cotidiana. Y entre los innumerables ele- 
mentos disponibles se encuentran los esquemas de tipificación o mapas cognoscitivos (Schutz, 1993; Dirven, 2001) que expresan la proximidad social de todo lo que hay al alcance e identifica a los integrantes de la tecnología social.

Este saber hacer proporciona un sentido de pertenencia y una forma de apreciar, lo cual pone límites a las previsiones que ordenan el porvenir, debido a que figura regularidades más o menos ciertas o virtualmente posibles, si los demás ya conocidos: terracerías, símbolos, objetos, artefactos, embalses, maquinarias, contratos, entidades divinas, canales de regadío, organizaciones, lugares físicos y esquemas de tipificación, por ejemplo, se comportan como tradicionalmente lo han hecho.

2. La tecnología social está situada en el tiempo histórico de una comunidad, y cada vez que se despliega dota de sentido al tiempo cronológico.

Esta cualidad puede ser entendida, en el marco de los procesos de modernización impulsados por el Estado nacional desarrollista, como la que contribuyó a disponer de un clima de época para lograr imaginar respuestas a las necesidades básicas cuando la política pública no cumplió sus objetivos de bienestar y de aumento de la calidad de vida, lo cual se produjo por la arremetida del Estado para reducir la heterogeneidad estructural de la base productiva de las sociedades en transición o en vía de desarrollo.

A continuación expongo una interpretación de García que nos permite introducirnos en las dimensiones que abarca esa tensión entre visiones y modos de concebir lo social.

De esta manera, destruida la ciudadanía sindical del Estado nacionalista, pero apenas asomada una nueva ciudadanía política moderna de tipo partidario y electivo, la sociedad ha empezado a crear o a retomar otras formas de mediación política, otras instituciones de ejercicio de representación, organización y movilización política, al margen de los partidos. Éstos son los nuevos, 
y viejos, movimientos sociales, con sus tecnologías de deliberación, del asambleísmo, cabildeo y acción corporativa, y de ahí que se pueda afirmar que, en términos de sistemas institucionales, hoy en Bolivia existen dos campos políticos. En regiones como el Chapare, Yungas y Norte de Potosí, la institucionalidad de comunidades se halla superpuesta no sólo a la organización partidaria, sino también a la propia institucionalidad estatal, en la medida en que alcaldes, corregidores y subprefectos están subordinados de facto a las federaciones campesinas. En el caso del altiplano norte, varias subprefecturas y puestos policiales provinciales han desaparecido en los últimos tres años, debido a las movilizaciones; en capitales provinciales se han creado "policías comunitarias", que resguardan el orden público en nombre de las Federaciones Campesinas y, de manera recurrente, cada vez que hay un nuevo bloqueo, cientos de comunidades altiplánicas erigen lo que ellas denominan el Gran Cuartel Indígena de Q'alachaca, que es una especie de confederación circunstancial de ayllus y comunidades en estado de militarización.

Ciertamente, todo ello tiene que ver con lo que alguna vez René Zavaleta denominó el "Estado aparente", en el sentido en que por la diversidad societal o civilizatoria del país, amplios territorios y numerosas poblaciones de lo que hoy denominamos Bolivia son portadores de formas de producir que no han interiorizado, como hábito y reforma técnica de los procesos laborales, la racionalidad capitalista, tienen otra temporalidad de las cosas, poseen otros sistemas de autoridad y de lo público, enarbolan fines y valores colectivos diferenciados a los que el Estado oferta como concepción del mundo y destino. Esto, que es una constante de la historia de los distintos estados bolivianos, hoy atraviesa procesos de autounificación institucional creciente, tanto coercitivos 
como simbólicos, bajo la forma de nacionalismos e identidades étnicas, que están dando lugar a una dualización de los sistemas políticos y principios de autoridad, en algunos casos de manera permanente (territorios agrario-indígenas politizados) y en otros, esporádicos (zonas urbanas de Cochabamba, La Paz y El Alto) (García, 2004:433-434).

En medio de los años setentas y como lo sostiene Humberto Ortiz, la crisis del paradigma no indígena empezó a ser evidente porque hubo una retirada de las confianzas en los modelos de crecimiento económico, y se inició el descredito en las tecnologías sociales que normalmente se reunían como modernas, formales y desarrolladas. Así esas tecnologías sociales comenzaron a significar todo lo contrario, lo cual no sólo se debe a la mala propaganda de la cual ya gozaban, sino que a un empalme entre el fracaso de su implementación en Latinoamérica y el fracaso de su reproducción en los países que debieron recuperarse de la Segunda Guerra Mundial, que lidiaron con la etapa de descolonización y que hoy intentan no dejar en un bote sin remos y a la deriva, a los ancianos y jóvenes de sus sociedades, mientras algunos elegidos hacen todo lo posible por desprenderse de sus responsabilidades y beneficiarse del esfuerzo colectivo.

3. La tecnología social se reproduce porque alguien la "organiza" y la "hace" "invitando" a los que simbólicamente se ubican como "responsables" en el esquema de tipificación que está a disposición de los que construyen un tiempo social, desde las evaluaciones sobre la trayectoria de la convivencia.

Un claro ejemplo de esta cualidad se manifiesta en un tipo de tecnología social que reconocemos con distinto nombre según el grupo o la sociedad estudiada; se trata de la "tanda" como la llaman en México (Lomnitz, 2003:94), "la polla" como la conocemos en Chile, pero también la encontramos bajo los nombres de "cundina, quiniela, mutualista, vaca". En Perú la identificamos con los nombres de "pandero" o "junta", en Guatemala: "chuchuval" 
y en los Estados Unidos: "tanda" o "cundina" (Vélez-Ibáñez, 1993:32-44). Esta tecnología social orientada al crédito, expresa el uso social de la cultura económica de los que la instituyen.

4. La tecnología social cuenta con tres encuentros ${ }^{11}$ elementales, a saber: observación, reconocimiento y resolución. En cada uno de ellos, los participantes arriban a un "consenso operativo" (Goffman Op. cit. en Wolf, 1979:36) donde se coordinan las miríadas de sentido atribuidos y se co-instituye el desenlace de la deliberación ${ }^{12}$.

Para que estos tres encuentros se desempeñen, es necesario que acontezcan interacciones donde se transfiera el saber hacer. Este tipo de interacciones descansan en dos dimensiones de la confianza, a saber: "expectativas sobre los encuentros" (VélezIbáñez, 1993:29) y "procesos de negociación" (Márquez, 2002:81) mediante los cuales se clausuran o reducen las incertidumbres dentro de los contextos pragmáticos.

5. La tecnología social presenta relaciones reglamentadas entre posiciones de cargos, entre ellos se encuentra el de "encargada" o "encargado", el cual se caracteriza porque aparece públicamente organizando y asume los mandatos de los que instituyen los encuentros colectivos.

Este cargo debe su posición a las siguientes cualidades: a) "conoce" la reputación de cada persona que cumple con los re-

11 Con la palabra encuentro, me hago parte de la concepción que Wolf rescata de la obra de Goffman (Wolf, 1979:30).

12 En palabras de Goffman, el asunto se describe de la siguiente manera: "presumiblemente una definición de la situación puede encontrarse siempre, pero los que están en la situación ordinariamente no crean tal definición []; normalmente todo lo que hacen es valorar correctamente lo que la situación debería ser para ellos $\mathrm{y}$, por lo tanto, actuar en consecuencia. Es verdad que personalmente negociamos aspectos de todas las circunstancias en las que vivimos, pero frecuentemente una vez que éstos están negociados continuamos mecánicamente como si las cosas hubiesen estado siempre ordenadas." (Goffman Op. cit. Wolf, 1979:39). En Bolivia esa deliberación aparece como "una institucionalidad basada en normas, procedimientos y culturas políticas tradicionales, corporativas no-liberales" (García, 2004:434). 
quisitos para ser "invitada" a la tecnología social, entre esas informaciones se encuentra, por ejemplo: el prestigio; b) la "encargada" es la que deberá "saber responder" por los actos de los integrantes de la tecnología social, c) revisa el estado de la tecnología social, d) administra un artefacto que "registra" los quehaceres de los que instituyen la tecnología social, y e) es la que inicia el ciclo de la tecnología social.

En el otro extremo de los cargos y posiciones. La tecnología social puede involucrar a las personas en cargos cuya posición supone el no conocimiento mutuo, o, pueden coexistir sin lograr gestar hitos de arraigo en la convivencia. Entonces, una persona puede incluirse sin que su participación defina el acumulado histórico de la tecnología social. Debido a esto, la tecnología social se desarrolla entre los que se hacen comunes en el tiempo social y en la distancia simbólica.

Esta variable es constatada por Vélez-Ibáñez, y la describe de la siguiente forma:

Los participantes no establecen el mismo grado de confianza con todos los miembros, pero se espera un mínimo de confianza entre el organizador y los participantes y entre algunos de los participantes. La confianza, entonces, se extiende a todos los demás participantes a través de varios vínculos.

Los vínculos de confianza serán tanto directos como indirectos y variarán en calidad y densidad. En muchos casos, los miembros deben confiar en la confianza de otros para completar sus obligaciones, dado que saben muy poco acerca de ellos. Tal como lo planteó un informante, "la confianza mutua se presta" (Vélez-Ibáñez, 1993:52).

En resumen, la "tecnología social" (Forni, 1988:59; Sen, 2000:68-74) es un arreglo normado de interacciones con tradición, y ella está inscrita o pertenece a las "redes sociotécnicas" (Latour, 2007:170-177). De esta forma, un territorio pueden ser concebido como el espacio social y el tiempo histórico donde es- 
tán varios tipos de tecnologías sociales que instituyen a las redes sociotécnicas que lo definen ${ }^{13}$.

\section{Comunidad}

En las presentaciones que escuchamos y en las indagaciones sobre el territorio y la tecnología social, hemos ido caracterizando a una sociedad popular desde el uso de sus posesiones simbólicas. Además, desde el inicio de esta reunión en la Universidad Federal de Rio de Janeiro, hemos supuesto, dentro y fuera de la sala -y yo no voy a impugnar ese consenso, sino que me pliego a su construcción-, que esa sociedad popular es un "mundo-devida" que expresa una "realidad histórica total que pertenece a un grupo social determinado, o una sociedad entera, en cuanto es vivida en la praxis del grupo y de sus miembros en todo lo que los identifica con el mismo grupo" (Moreno, 2006:45).

A partir de ahí y recordando el énfasis que proponen Beatriz, Patricio y Humberto, sobre una integración en la diversidad, es posible sostener que hay una coexistencia de mundos de vida. Y, en algunos casos, uno de esos mundos trata de imponerse y otros viven al margen. Entonces es posible suponer que el "modo de

13 En el trabajo de Herrera que en esta oportunidad sólo utilizo de forma anexa, él establece una definición que emerge desde el estudio que realiza sobre las tecnologías andinas, bajo el encuentro virtuoso de la Arqueología con la Antropología y que también comparte lo que en esta jornada los expositores han ido mostrándonos.

Herrera plantea su definición de la siguiente forma: "Las tecnologías indígenas andinas conforman un conjunto de prácticas históricamente enraizadas que posibilitan sistemas de manejo de un entorno diverso, complejo y transformado, enmarcado en formas culturales particulares de ver y entender el mundo. Como Lemmonier (1993) y otros han recalcado, la tecnología es irreducible a un conjunto de 'cosas', 'saberes' o 'maneras de hacer' divorciados de su contexto social, histórico y cultural específico. La tecnología puede ser entendida como una fuerza abstracta que involucra los aspectos económicos y políticos de los objetos y su uso productivo, tanto como sus facetas sociales, religiosas o culturales. Es en este sentido que la definimos como un conjunto de prácticas sociales, imbricado en redes sociales tejidas alrededor de objetos, lugares en el paisaje y conocimientos culturales específicos." (Herrera, 2011:135) 
Tecnologías Sociales y Economía Solidaria. Una revisión desde las realidades nacionales marcadas por la presencia de pueblos originarios - Nicolás Gómez

vida popular" ha estado "desacoplado del sistema dominante", pero esta situación no lo sitúa como un subsistema de esa matriz que "distingue estructuras formales de integración" en un "consenso cultural". Esto, debido a que ese modo de vida popular se desarrolla "fuera de sus márgenes" y donde su "actividad" no le permite percibirse como "marginado" (Moreno, 2006:45).

Ese obrar colectivo que despliega su propia trayectoria, es "una comunidad cultural diferenciada y un proyecto político" (García, 2004:430) que cuenta con un horario oficial donde se ponen en marcha los complejos de tecnologías sociales, o se detienen sus usos eficientes. En resumidas cuentas, gobierna el tiempo socialmente aceptado en el marco de una "economía campesina semimercantil" (García, 2004:431), y esa conquista colectiva les brinda un sentido de pertenencia porque ofrece los hitos de arraigo del nosotros. Por ejemplo, la seguridad ontológica que compromete a las personas en el obrar colectivo que impiden el cálculo y fomenta una praxis ${ }^{14}$ espontánea, irreflexiva e inintencionada que reproduce el sentido de integración del mundo de vida (Moreno, 2006:48). Y, nosotros, al observar la dignidad de esa autoridad, reconocemos ahí una comunidad que co-existe hilvanando la historia.

Esta manera de abordar el asunto reviste importancia práctica y analítica, si constatamos que los programas implementados tanto por los Estados, especialmente los de Bolivia y Ecuador, como por las organizaciones no gubernamentales, han avanzado incorporando a los beneficiarios, o población objetivo, como sujetos de derechos económicos, sociales y culturales, lo que ha permitido dejar de apreciarlos como carentes de capacidades y se los ha asumido como los principales gestores de las maneras que les ayudan a dar respuesta a su situación.

14 Un elemento clave de este "mundo-de-vida" es la noción de praxis, y Moreno la asume como "el ejercicio mismo de practicar el vivir que ejerce una comunidad humana en un tiempo histórico determinado, que puede ser de muy larga duración, y del que participa espontáneamente cada uno de sus miembros" (Moreno, 2006:48). 
En esta perspectiva, asumir a la comunidad en su dignidad y validar su saber hacer, no sólo facilita explicar el desarrollo local desde sus bases económicas (González, 1993:226-227), sino que también provoca una ruptura en la concepción de la protección social en tanto se hace relevante la sociabilidad como recurso que da acceso a las redes (Márquez, 1993:79) donde se reproducen las representaciones sociales y los sistemas de comunicación (Castells, 1999:111), y ayuda a la "gestión territorial de lo social" (González, 1993:225).

Sin embargo, ese estado de seguridad en el mundo de vida de la comunidad popular, no clausura las deliberaciones que las personas, las familias y las organizaciones pueden hacer sobre su matriz cultural, en torno a los sentidos atribuidos a lo social o sobre los usos de las informaciones sociotécnicas que están en el saber hacer de las tecnologías sociales; pero sí limita la ocurrencia de las deliberaciones que interrogan sobre la ubicación de esa sociedad popular en el concierto universal, gracia a lo cual la vida, esa vida, transcurre como la única posibilidad mediata.

Esto trae consigo algunas consecuencias. Por ejemplo, la dignidad del obrar colectivo sostiene a las personas para construir lo público; en esos términos se produce la educación en la ética de la conducta y especialmente en los criterios que se deben tomar siempre en cuenta para conducirse pertinentemente, para lograr co-instituir respuestas a los problemas o satisfacer las necesidades comunitarias ${ }^{15}$. Por tanto, la competencia de las personas está

15 En las reflexiones que realiza Herrera, lo que he señalado puede tener la siguiente expresión: "Las estrategias andinas tienden a ser agrosilvopastoriles y buscan maximizar la distribución de riesgos en el tiempo y el espacio, por lo que se traslapan con el aprovechamiento 'vertical' de pisos ecológicos que caracteriza las áreas montañosas del mundo en general, y de los Andes en particular. Es necesario reconocer la importancia de las trayectorias históricas de regulación autogestionaria de la distribución de agua, generalmente -mas no siempre- organizada en los grupos de comunidades que comparten cuencas particulares y vinculadas a ciclos festivos y de trabajo, por encima de los derechos de propiedad privados sobre el agua o la tierra o la intervención del Estado. Este punto es fundamental, en cuanto 
Tecnologías Sociales y Economía Solidaria. Una revisión desde las realidades nacionales marcadas por la presencia de pueblos originarios - Nicolás Gómez

basada en su participación pertinente según el uso de los criterios éticos que definen las responsabilidades individuales en los esfuerzos colectivos que despliegan estos tipos de obrares. Sin embargo, no debería interpretarse esta disposición a hacer comunidad como la regular tendencia a incluir todo lo que se encuentre a mano dentro de lo que desde ahí se define como el nosotros. Parece altamente probable reconocer fronteras que diferencian al menos dos tipos de individuos $\mathrm{u}$ organizaciones.

Uno de esos tipos permite clasificar a las personas y sus formas asociativas, dentro de aquellas con las cuales es posible comprometerse, fijar el devenir y reproducir las reciprocidades. En este tipo ideal pueden reunirse las personas cuyas relaciones presentan una "cohesión técnica" (García, 2009: 272). Por el contrario, en el otro tipo quedan situados los individuos y sus organizaciones, que han fracturado los compromisos o que no cuentan con la reputación de crédito y credibilidad. Por lo cual, se fija un derecho a negar la posibilidad de participar en la tecnología social o, en términos generales, en los beneficios del territorio.

Esto nos plantea dos situaciones. La primera, es posible que una organización o una persona que sea marginada pueda tener una existencia en la convivencia vecinal pero no en la tecnología social, debido a que sus relaciones de parentesco y las de habitar históricamente una vecindada, impiden su expulsión. La segunda posibilidad es la expulsión de una persona u organización que ha llegado a cohabitar en el territorio, pero que su conducta expresa una ética que la transforma en un mal vecino que promueve la fractura de la convivencia.

la inversión de trabajo necesaria para poner en funcionamiento infraestructura productiva abandonada o arqueológica requiere de una visión de conjunto a largo plazo, compartida por todos los actores imbricados en la labor y fundamentada en una evaluación cualitativa de la relación entre los seres humanos y su entorno, que ponga las generaciones futuras por delante de la rentabilidad económica coyuntural, es decir, la sustentabilidad ecológica y cultural de las técnicas empleadas, por delante de las doctrinas del desarrollo." (Herrera, 2011:135) 


\section{Conclusiones}

Es posible reconocer que la noción de territorio está definida por quien organiza la mirada sobre el presente. Al revisar el asunto desde América Latina, especialmente desde las sociedades de Los Andes, esa idea abarca la proximidad que sostiene a las comunidades que producen bienes y servicios de uso colectivo, y a los tiempos sociales de los contenidos densos que orientan las relaciones de cooperación y distribución.

Por otra parte, debemos explorar el saber que ya existe sobre las tecnología sociales, necesitamos documentar los arreglos normados de las interacciones con tradición que han facilitado, aún en los peores tiempos, la reproducción de las sociedades latinoamericanas; y requerimos diseñar políticas públicas que vinculen a esas tecnología y sus comunidades, con las universidades y el Estado para promover la diversificación de sus redes sociotécnicas. Porque el asunto central es fijar un recorrido orientado por un modelo de buen vivir, debido a que permiten la integración en base al trabajo que instituye lo público desde la relación entre la familia, la comunidad y la naturaleza.

A continuación cierro mis comentarios y agradezco la posibilidad que me ha brindado SOLTEC-UFRJ, por tener una cálida acogida y así conocer las reflexiones de Beatriz Rodríguez, Patricio Andrade y Humberto Ortiz. Además, quiero agradecer al Ministerio del Trabajo de Brasil y a su Secretaría Nacional de Economía Solidaria, por mostrarme una forma de hacer política pública y por la gestión de las alianzas que han hecho posible tenernos en esta universidad, lo cual demuestra la claridad de las convicciones de quienes están actualmente en el gobierno. Finalmente, no quiero dejar pasar la oportunidad de felicitar a las y los colegas que participan en la Red de Investigadores Latinoamericanos de Economía Social y Solidaria (RILESS), porque gracias a su empeño, hoy muchas personas avanzan en estas materias. 
Tecnologías Sociales y Economía Solidaria. Una revisión desde las realidades nacionales marcadas por la presencia de pueblos originarios - Nicolás Gómez

\section{Bibliografía}

BAUMAN, Z. (2003): Comunidad. En busca de seguridad en uin mundo hostil, Buenos Aires, Siglo XXI.

BERIAN, J. (com.) (1996): Las consecuencias perversas de la modernidad, Barcelona, Anthropos.

BOISIER, S. (2001): Desarrollo (Local): ¿De qué estamos hablando? En A. Vásquez y O. Madoery (comps.), Transformaciones globales, instituciones y políticas de desarrollo local (pp. 4874). Rosario, Argentina : Homo Sapiens.

BOURDIEU, P. (2006): Argelia 60. Estructuras económicas y estructuras temporales, Buenos Aires, Siglo XXI.

CASTELLS, M. (1999): La cuestión urbana, México D.F., Siglo Veintiuno.

CASTELLS, R. (2002): “¿Qué significa estar protegidos?”, en DABAS, E. et al., Redes. El lenguaje de los vínculos. Hacia la reconstrucción y el fortalecimiento de la sociedad civil, Buenos Aires, Paidós; 151-158.

DIRVEN, M. (2001): Entre el Ideario y la Realidad: Capital Social y Desarrollo Agrícola, Santiago de Chile, mimeografiado.

DURSTON, J. (2002): Capital social: definiciones, controversias, tipologías. En CEPAL (Eds.). El capital social campesino en la gestión del desarrollo rural (pp. 1-8). Chile: CEPAL.

FORNI, F. (1992): Formulación y evaluación de proyectos de acción social, Buenos Aires: Humanitas.

FORNI, F.; BENENCIA, R. y NEIMAN, G. (1991): Empleo, Estrategias de vida y Reproducción. Hogares rurales en Santiago del Estero, Buenos Aires, Centro Editor de América Latina.

GARCÍA, J. (1994): La racionalidad en política y Ciencias Sociales, Buenos Aires, Centro Editor de América Latina.

GARCIA, Á. (2004): “Crisis del Estado y sublevaciones indígena-plebeyas en Bolivia", en Álvaro García Linera, Luis Tapia y Raúl Prada, Memorias de octubre, La Paz, Comuna y Muela del Diablo, 2004.

GARCÉS, M. (1997): Historia de la comuna de Huechuraba. Memoria y oralidad popular urbana. Santiago, ECO, Educación y Comunicaciones.

GARRETÓN, M. (1990): Las transiciones a la democracia y el caso chi- 
leno, en Iglesia y transición en Chile (pp. 17-28). Serie de estudios del CEDM, nº 4, Santiago, Rehue.

GODELIER, M. (1996): El enigma del don, Buenos Aires, Paidós.

GOFFMAN, E. (2006): Estigma. La identidad deteriorada, Buenos aires, Arorrortu.

GONZÁLEZ, R. (1993): Desarrollos productivos locales y pequeña producción, en Proposiciones $n^{\circ} 23$ : Microempresa y desarrollo (pp. 218-232), Santiago, Sur.

GREZ, S. y SALAZAR, G. (com) (1999): Manifiesto de Historiadores. Santiago, Lom.

HARDY, C. (1985): Hambre + dignidad = Olla común, Santiago, PET.

HERRERA, A. (2011): La recuperación de tecnologías indígenas. Arqueología, tecnología y desarrollo en Los Andes, Lima, Instituto de Estudios Peruanos, Universidad de los Andes, Facultad de Ciencias Sociales-CESO, Departamento de Antropología; Consejo Latinoamericano de Ciencias Sociales, PUNKU Centro de Investigación Andina.

LATOUR, B. (2007): Reensamblar lo social. Una introducción a la teoría del actor-red, Buenos Aires, Manantial.

LOMNITZ, L. (2008): Lo formal y lo informal en las sociedades contemporáneas, Santiago, centro de investigaciones Barros Arana.

(2003): Cómo sobreviven los marginados, Buenos Aires, Siglo XXI.

MORENO, A. (2006): El aron y la trama. Episteme, Modernidad y Pueblo, Santiago: Universidad Católica Silva Henríquez.

PASTOR, A.; MENA, S.; ROJAS, S. y PARRAGUEZ, W. (1993): De Lonquén a Los Andes, Santiago, Rehue.

PERRET, B. y ROUSTANG, G. (2000): La economía contra la sociedad. Crisis de integración social y cultural, Santiago, Fondo de Cultura Económica.

SALAZAR, G. (2000): Labradores, Peones y Proletarios, Santiago, LOM. (2006): Ser niño "huacho" en la historia de Chile (siglo XIX), Santiago, LOM.

SEN, A. (2000): "Género y conflictos cooperativos", en Navarro, Marysa: Cambios sociales, económicos y culturales, Buenos Aires, Fondo de Cultura Económica. 
Tecnologías Sociales y Economía Solidaria. Una revisión desde las realidades nacionales marcadas por la presencia de pueblos originarios - Nicolás Gómez

SCHÜTZ, A. (1993): La construcción significativa del mundo social, Buenos Aires, Paidós.

VÉLEZ-IBÁÑEZ, C. (1993): Lazos de confianza. Los sistemas culturales y económicos de crédito en las poblaciones de Estados Unidos y México, México D. F., Fondo de Cultura Económica.

WACQUANT, L. (2007): Parias urbanos. Marginalidad en la ciudad a comienzos del milenio, Buenos Aires, Manantial.

WOLF, Mauro (1979): Sociologías de la vida cotidiana, Madrid: Cátedra. 